\title{
SPH simulations of star/planet formation triggered by cloud-cloud collisions
}

\author{
Spyridon Kitsionas ${ }^{1}$, Anthony P. Whitworth ${ }^{2}$ and Ralf S. Klessen ${ }^{3}$ \\ ${ }^{1}$ Astrophysikalisches Institut Potsdam, An der Sternwarte 16, D-14482 Potsdam, Germany \\ email: skitsionas@aip.de \\ ${ }^{2}$ School of Physics \& Astronomy, Cardiff University, P.O. Box 913, CF24 3AA Cardiff, U.K. \\ ${ }^{3}$ Institut für Theoretische Astrophysik, Universität Heidelberg, \\ Albert-Ueberle-Str. 2, D-69120 Heidelberg, Germany
}

\begin{abstract}
We present results of hydrodynamic simulations of star formation triggered by cloudcloud collisions. During the early stages of star formation, low-mass objects form by gravitational instabilities in protostellar discs. A number of these low-mass objects are in the sub-stellar mass range, including a few objects of planetary mass. The disc instabilities that lead to the formation of low-mass objects in our simulations are the product of disc-disc interactions and/or interactions between the discs and their surrounding gas.
\end{abstract}

Keywords. hydrodynamics, methods: numerical, stars: formation, planetary systems: protoplanetary disks, planetary systems: formation

\section{Introduction}

In the classical picture (Shu, Adams \& Lizano 1987) stars form from the collapse of a single gaseous core in isolation: all stages of the star formation process (i.e. objects of Class 0, I, II, III) as well as planet formation are terminated before the newly formed star/planetary system interacts with its environment (e.g. other stars/planetary systems, and/or its surrounding gas). In the more dynamic star formation paradigm of gravoturbulent fragmentation (Mac Low \& Klessen 2004) interactions during the formation process are not only a common phenomenon but also a sufficient condition for the formation of binary and higher-order multiple systems, as well as for explaining the shape of the Initial Mass Function (Jappsen et al. 2005; see also Bate, Bonnell \& Bromm 2003, Bate \& Bonnell 2005), the evolution of the angular momentum distribution of protostars (Jappsen \& Klessen 2004), their mass accretion rates (Schmeja \& Klessen 2004), the spatial distribution of young stellar objects (Schmeja \& Klessen 2006), etc. The competing paradigm of a more isolated star formation process advocated by Krumholz \& McKee (2005), uses large-scale turbulent support against collapse in a rather isotropic way, giving rise to supercritical cores with a variety of masses (see e.g. Krumholz, McKee \& Klein 2005 vs. Bonnel \& Bate 2006). In contrast, gravoturbulent star formation is based on the small-scale density fluctuations produced by supersonic turbulence, with gravity then promoting the strongest of these fluctuations, determining the onset of collapse.

In the context of gravoturbulent fragmentation, Chapman et al. (1992), Turner et al. (1995), Whitworth et al. (1995), and Bhattal et al. (1998) have studied star formation triggered by cloud-cloud collisions. In essence, the cloud-cloud collision mechanism describes the small-scale evolution of the interaction between colliding flows (VázquezSemadeni et al. 2006, 2007). We note that several authors have studied the interaction between large-scale colliding flows in non-self-gravitating media, discussing the importance of such interactions for the formation of molecular-cloud structure and turbulence 
(e.g. Hunter et al. 1986, Walder \& Folini 1998, Heitsch et al. 2005, Hennebelle \& Audit 2007). These authors discuss the formation at best of low-mass structures such as dense cloud cores, whereas in our self-gravitating simulations we use such dense cores as the initial conditions in order to follow star formation.

In this paper we present the results of recent high-resolution hydrodynamic simulations of collisions between low-mass clumps (Kitsionas \& Whitworth 2007). Due to the high numerical resolution of our simulations, we can resolve, for the first time in simulations of cloud-cloud collisions, the formation of sub-stellar objects, some of which have a mass that lies close to the boundary between brown dwarves and planetary-mass objects, i.e. $\sim 15 M_{J}$. These planetary-mass objects form by gravitational instabilities in the disc of their parent protostar, i.e. by the same mechanism that is responsible for the formation of low-mass stellar and/or brown dwarf companions to the central protostar of such discs. Moreover, the disc instabilities that lead to the formation of low-mass objects in our simulations are the product of disc-disc interactions and/or interactions between the discs and their surrounding gas. Furthermore, we deal here with disc instabilities of young, massive discs still in the process of formation, i.e. at the early stages of the disc life. We therefore caution the reader not to confuse the instabilities reported here with the extensive work on gravitational instabilities and related phenomena in thin, or in general evolved discs that have been recently reviewed by Durisen et al. (2007). As for the core accretion model of planet formation, we refer the reader to a number of papers in this volume dealing with the different stages of solid core formation, gas accretion and migration in protoplanetary discs.

In section 2, we give a brief description of the model and the numerical method we use. In section 3, we present our results. In section 4 , we discuss our findings with respect to models of gas giant planet formation due to gravitational instabilities in protostellar discs.

\section{Our model and numerical method}

In Kitsionas \& Whitworth (2007), we investigate, by means of numerical simulations, the phenomenology of star formation triggered by low-velocity collisions between lowmass molecular clumps. The simulations are performed using a smoothed particle hydrodynamics (SPH) code which satisfies the Jeans condition by invoking on-the-fly Particle Splitting (Kitsionas \& Whitworth 2002).

Clumps are modelled as stable truncated (non-singular) isothermal, i.e. Bonnor-Ebert, spheres. Collisions are characterised by $M_{0}$ (clump mass), $b$ (offset parameter, i.e. ratio of impact parameter to clump radius), and $\mathcal{M}$ (Mach Number, i.e. ratio of collision velocity to effective post-shock sound speed). The gas follows a barotropic equation of state, which is intended to capture (i) the scaling of pre-collision internal velocity dispersion with clump mass (Larson 1981), (ii) post-shock radiative cooling, and (iii) adiabatic heating in optically thick protostellar fragments (with exponent $\gamma \simeq 5 / 3$ ). The equation of state we use is given by Eq. 1 of Kitsionas \& Whitworth (2007) and is graphically illustrated in the temperature-density plane by their Fig. 1. In short, the initial effective isothermal sound speed of the gas, i.e. when non-thermal pressure due to turbulence is included, follows the Larson (1981) relations in order to model the observed variation of the internal velocity dispersion with clump mass. As long as the collisions begin, the effective sound speed is reduced according to a $P \propto \rho^{1 / 3}$ relation until it reaches a value equivalent to the temperature of $10 \mathrm{~K}$. Then the gas remains isothermal with increasing density up to the point where it becomes opaque to its own cooling radiation and heats up as $P \propto \rho^{5 / 3}$. 

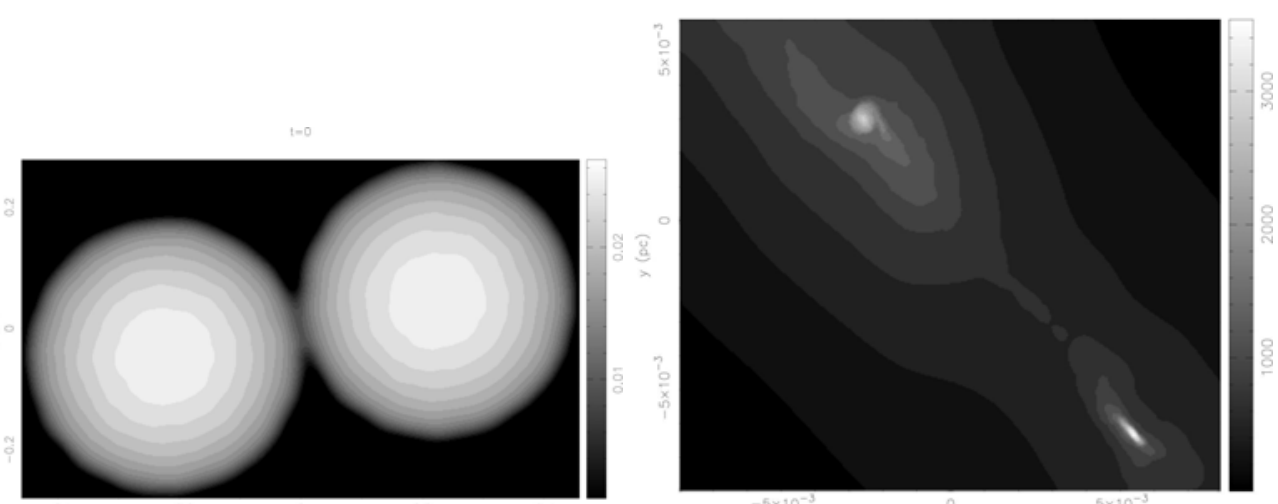

Figure 1. Column density plots for the collision with $M_{0}=10 M_{\odot}, b=0.2, \mathcal{M}=10$. Left Panel. Initial conditions viewed along the $z$-axis; $\Delta x=0.92 \mathrm{pc}, \Delta y=0.56 \mathrm{pc}$; the grey-scale is logarithmic, in units of $\mathrm{g} \mathrm{cm}^{-2}$, with sixteen equal intervals from $1.00 \times 10^{-3} \mathrm{~g} \mathrm{~cm}^{-2}$ to $2.69 \times 10^{-2} \mathrm{~g} \mathrm{~cm}^{-2}$. Right Panel. $t=0.396 \mathrm{Myr}$; viewed along the $z$-axis; $\Delta x=0.016 \mathrm{pc}, \Delta y=0.014 \mathrm{pc}$; sixteen-interval logarithmic grey-scale, in units of $\mathrm{g} \mathrm{cm}^{-2}$, from $2.14 \times 10^{-1} \mathrm{~g} \mathrm{~cm}^{-2}$ to $3.55 \times 10^{3} \mathrm{~g} \mathrm{~cm}^{-2}$.

From the variety of models we have investigated, we present here the collision between two $M_{0}=10 M_{\odot}$ clumps, each moving with velocity (along the $x$-axis) $v_{\text {clump }}=1 \mathrm{~km}$ $\mathrm{s}^{-1}$ (corresponding to a Mach number $\mathcal{M}=10$, i.e. assuming a post-shock sound speed of $0.2 \mathrm{~km} \mathrm{~s}^{-1}$ ), and with an offset parameter $b=0.2$ taken along the $y$-axis. The initial conditions are shown in the left panel of Fig. 1.

We evolve the simulations using an SPH code. This is a Lagrangian method for 3D hydrodynamics. In SPH the sampling points (particles) are able to move with the fluid and their properties are distributed in space through a smoothing function that allows all hydrodynamic quantities to be continuous (Monaghan 1992). Our self-gravitating SPH code (Turner et al. 1995) uses tree-code gravity (Barnes \& Hut 1986) to reduce the computational cost for the calculation of gravitational forces, and a second order RangeKutta time integrator with multiple particle timesteps. It also uses Particle Splitting (Kitsionas \& Whitworth 2002), which allows the on-the-fly increase of numerical resolution (in terms of splitting the SPH particles) when and where this becomes necessary, i.e. every time that violation of the Jeans condition (Bate \& Burkert 1997, Truelove et al. 1997) becomes imminent. The benefit of the use of Particle Splitting is twofold. Firstly, gas fragmentation remains resolved at all times. Secondly, the simulations evolve with minimum computational cost, as high numerical resolution is employed only when required. Our Particle Splitting method assigns velocities to new particles by interpolating over the velocity field of their parent particle population, thus achieving conservation of energy and momenta with very high accuracy.

Our code includes sink particles (Bate, Bonnell \& Price 1995). These are collisionless (star) particles that replace dense, collapsed gaseous regions in order to allow the simulations to evolve further in time. With sink particles, the simulations avoid using tiny timesteps to follow the evolution internal to the sink but at the same time all information on scales smaller than the size of the sink particle becomes invisible to us. In this work we use sink particles with radii $\sim 20$ AU. A summary of all features of our code can be found in Kitsionas \& Whitworth (2007). 

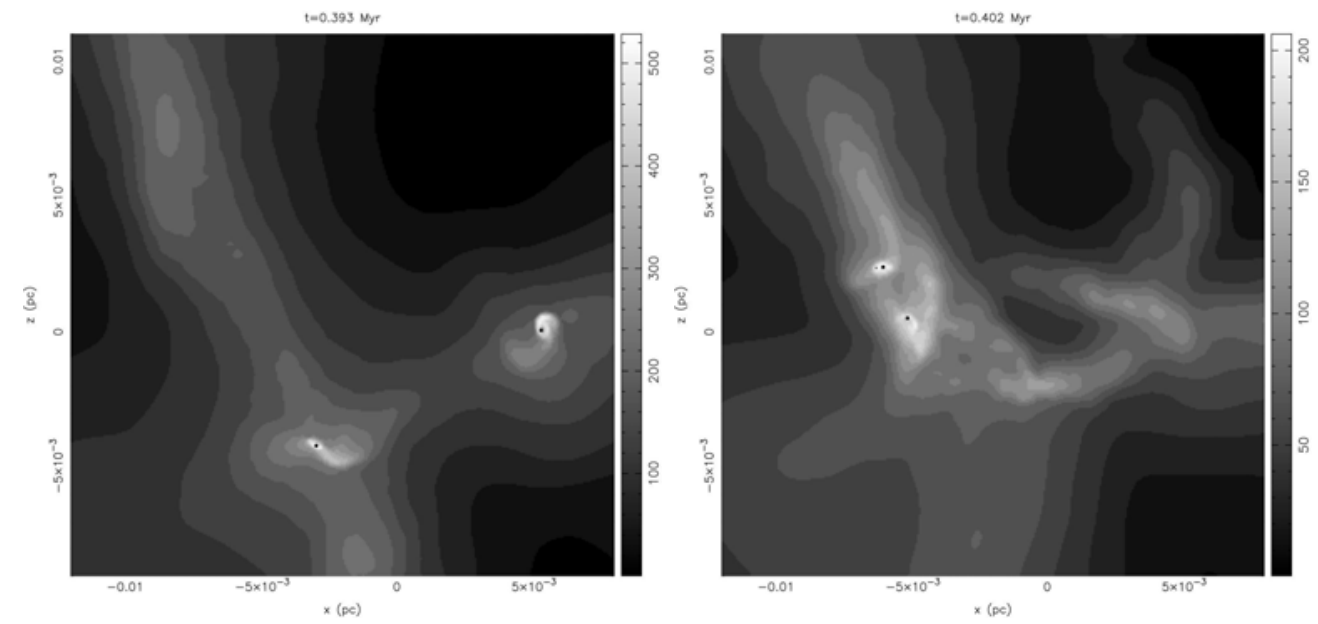

Figure 2. Column density plots for the collision with $M_{0}=10 M_{\odot}, b=0.2, \mathcal{M}=10$, with sink particles overlayed as solid circles (note that the symbol used for the sinks is larger than the actual sink radius at the scale of these plots). View along the $y$-axis; $\Delta x=\Delta z=0.02 \mathrm{pc}$ in both panels. Left Panel. $t \sim 0.393 \mathrm{Myr}$, i.e. very close to the time of the right panel of Fig. 1; sixteen-interval logarithmic grey-scale, in units of $\mathrm{g} \mathrm{cm}^{-2}$, from $3.31 \times 10^{-1} \mathrm{~g} \mathrm{~cm}^{-2}$ to $5.25 \times 10^{2} \mathrm{~g} \mathrm{~cm}^{-2}$. Right Panel. $t \sim 0.402 \mathrm{Myr}$; sixteen-interval logarithmic grey-scale, in units of $\mathrm{g} \mathrm{cm}^{-2}$, from $2.88 \times 10^{-1} \mathrm{~g} \mathrm{~cm}^{-2}$ to $2.04 \times 10^{2} \mathrm{~g} \mathrm{~cm}^{-2}$.

\section{Results}

A shock-compressed layer forms at the collision interface as the collision proceeds. The right panel of Fig. 1 offers a zoom-in view of this layer at $t \sim 0.396 \mathrm{Myr}$. The layer extends along the $z$-axis. The $x$ - $z$ projection of the shock-compressed layer at this time is shown in the left panel of Fig. 2. The layer has broken up into filaments and the filaments have fragmented into protostars surrounded by circumstellar discs. Sink particles have replaced the central protostars of these discs. A second protostar forms on the upper part of the filament that extends almost vertically in this panel. At $t \sim 0.402 \mathrm{Myr}$ (right panel of Fig. 2) the two protostars in this filament (represented by sink particles surrounded by circumstellar discs) have moved along the filament towards each other. Due to the tumbling nature of the filament, they have a close encounter in the course of which they capture each other into a binary. During this interaction, their discs merge to form a circumbinary disc (left panel of Fig. 3). This disc-disc interaction leads also to the fragmentation of the circumbinary disc (see the additional sink particles in this panel). At later times, the circumbinary disc fragments only when lumps of material from the filament fall on to the disc (e.g. see the lumps on the right panel of Fig. 3).

Each of the low-mass fragments, which form during the disc-disc interaction and/or during the episodes of lumpy accretion from the filament, gets ejected from the system after a close encounter with the binary. The final state of the system (Fig. 4) includes a large circumbinary disc hosting a binary, with components having mass $\sim 1.5 M_{\odot}$ (each) and separation $\lesssim 40 \mathrm{AU}$, as well as a number of low-mass objects that have been ejected from the disc with moderate velocities of order a few $\mathrm{km} \mathrm{s}^{-1}$. Some of these low-mass objects are sub-stellar or even of planetary mass. After their ejection from the circumbinary disc they have stopped accreting more mass. 

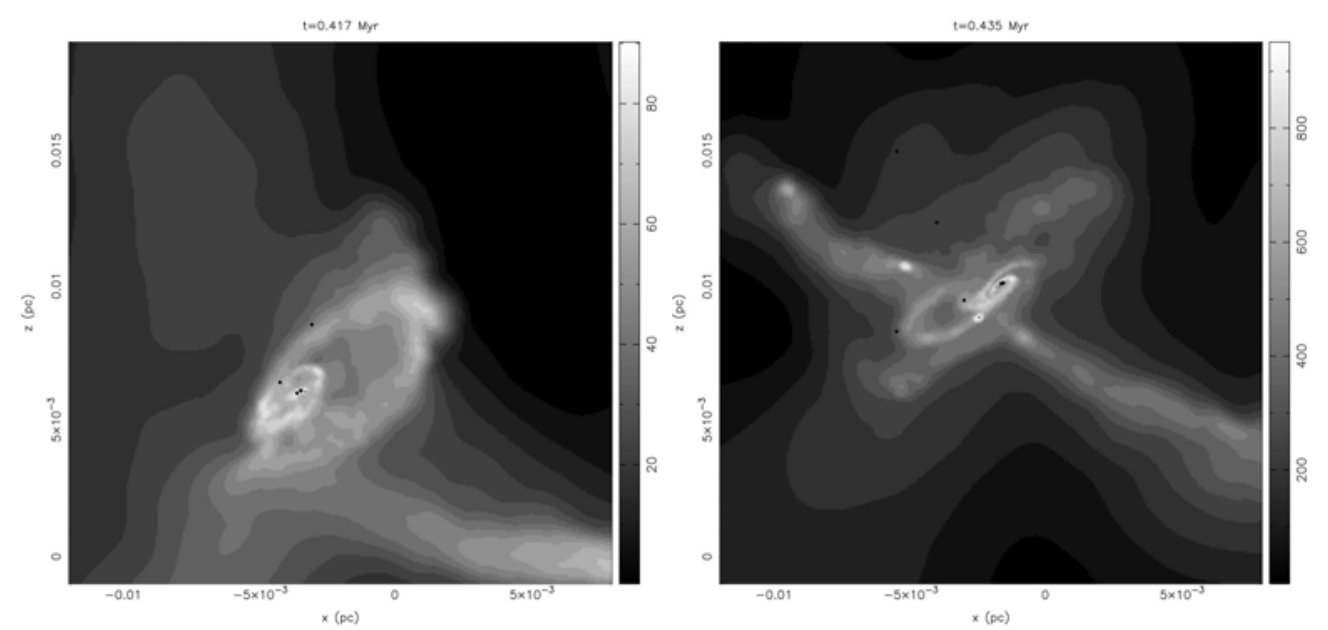

Figure 3. Column density plots for the collision with $M_{0}=10 M_{\odot}, b=0.2, \mathcal{M}=10$, with sink particles overlayed as solid circles (note that the symbol used for the sinks is larger than the actual sink radius at the scale of these plots). View along the $y$-axis; $\Delta x=\Delta z=0.02 \mathrm{pc}$ in both panels (note that these panels have been shifted by $0.008 \mathrm{pc}$ along the $z$-axis with respect to the panels of Fig. 2). Left Panel. $t \sim 0.417 \mathrm{Myr}$; sixteen-interval logarithmic grey-scale, in units of $\mathrm{g} \mathrm{cm}^{-2}$, from $2.45 \times 10^{-1} \mathrm{~g} \mathrm{~cm}^{-2}$ to $9.12 \times 10^{1} \mathrm{~g} \mathrm{~cm}^{-2}$. Right Panel. $t \sim 0.435 \mathrm{Myr}$; sixteen-interval logarithmic grey-scale, in units of $\mathrm{g} \mathrm{cm}^{-2}$, from $3.09 \times 10^{-1} \mathrm{~g} \mathrm{~cm}^{-2}$ to $9.55 \times 10^{2} \mathrm{~g} \mathrm{~cm}^{-2}$.

\section{Discussion}

Based on the results presented here, we can claim with confidence that dynamical interactions during the early stages of star formation provide a mechanism for the formation of low-mass objects. Disc-disc interactions and/or interactions between the discs and their gaseous environment (e.g. episodes of lumpy accretion from a filament) can lead to gravitational instabilities in protostellar discs and the formation of low-mass objects. Some of these objects may subsequently be ejected from the disc they formed in. As a result of this ejection, their accretion rates will drop significantly and they will end-up in the sub-stellar or even planetary-mass regime.

It is important to note that the gas in our discs is rather warm, as no cooling mechanism for discs is included in our code and adiabatic gas heating has already switched on at the gas densities of our discs (see section 2 for a brief description of our equation of state). Therefore, the gravitational instabilities we report here are due to an increase in the disc surface density (caused by a dynamical interaction) and not because of the decrease of the gas sound speed in the disc.

We note that the discs in our simulations contain only a few thousand SPH particles and evolve for a small number of orbital periods. Thus, due to excess numerical dissipation and limited orbital evolution, we are not yet in a position to investigate in detail the angular momentum evolution of gas and fragments in our discs. Nevertheless, fragmentation here is mostly externally triggered and therefore we are confident that it is not due to spurious angular momentum transport. Moreover, since the discs form self-consistently in a cluster environment, it is inevitable that during the initial stages of their formation they will contain a small but increasing number of particles. This makes impossible to comment on the effect of numerical dissipation on early disc evolution. Convergence studies with respect to the disc numerical dissipation in our simulations are currently underway. 


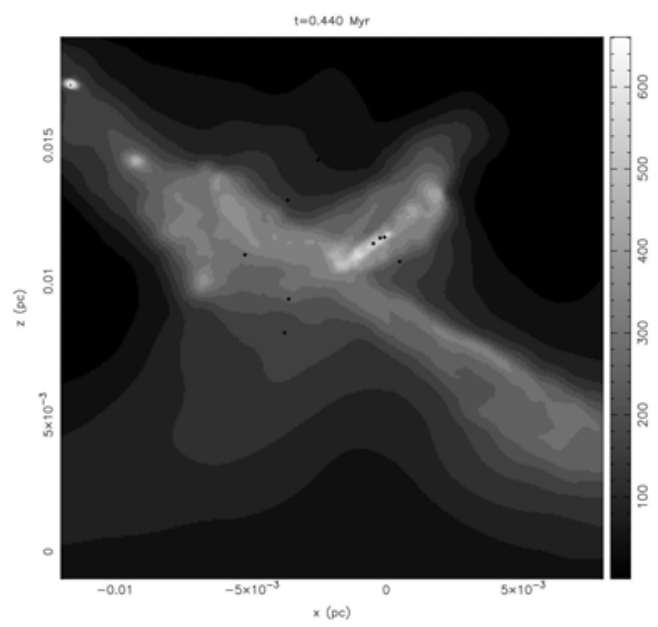

Figure 4. Column density plots for the collision with $M_{0}=10 M_{\odot}, b=0.2, \mathcal{M}=10$, with sink particles overlayed as solid circles (note that the symbol used for the sinks is larger than the actual sink radius at the scale of these plots). The final state of the simulation $(t \sim 0.440 \mathrm{Myr})$; view along the $y$-axis; $\Delta x=\Delta z=0.02 \mathrm{pc}$; sixteen-interval logarithmic grey-scale, in units of $\mathrm{g} \mathrm{cm}^{-2}$, from $3.39 \times 10^{-1} \mathrm{~g} \mathrm{~cm}^{-2}$ to $6.61 \times 10^{2} \mathrm{~g} \mathrm{~cm}^{-2}$.

Gas cooling is an important ingredient in simulations of isolated protostellar discs (e.g. see Rice et al. 2003) even in the case that this cooling is not sufficient to produce gravitational instabilities and fragmentation of the disc (see the discussion in Durisen et al. 2007).

Stamatellos, Hubber \& Whitworth (2007) have recently reported gravitational instabilities that lead to the formation of low-mass objects in an isolated disc using an SPH code with on-line (approximate) radiation transport. Their radiation hydrodynamics method is effective in calculating disc cooling close to the disc midplane but not further out in the disc "atmosphere". We are currently developing an improved algorithm that will be able to identify the scale-height at which each gas particle lies and thus assign the cooling efficiency of the particle accordingly.

Generalising our finding on the importance of dynamical interactions during the early stages of star formation, we assert that such interactions may be important for the formation of low-mass objects also at later stages of protostellar evolution, as long as a gas disc is still present. The outcome of such interactions need not be free-floating objects in all cases, as in less "chaotic" situations (e.g. in circumstellar discs around single stars) the low-mass objects formed may remain bound to the system they formed in. Moreover, interactions of already formed planetary systems with incoming stars or lower mass freefloating objects may disturb the planetary systems, but this is beyond the scope of this paper (see several papers in this volume on planetary system dynamics).

Taking our results at face value, one can claim that the majority of the low-mass objects forming in such simulations will end up unbound from the system of their birth, i.e. a number of free-floating objects are expected to form as a by-product of star formation.

The formation of free-floating planetary-mass objects is of particular interest. Such objects can be observed mainly through their gravitational signature. In the field, this signature could be detected only through microlensing observations. Zinnecker (2001) has calculated that a lensing planet can produce a microlensing event with duration of a few days. He has also estimated that one such event is expected out of $3 \times 10^{8}$ observed lightcurves. He has further advocated that campaigns for the observation of free-floating 
planetary-mass objects through microlensing can become possible when the VST and VISTA facilities start their operation as well as with the JWST.

The event probability and duration that Zinnecker (2001) has estimated are based on simple calculations involving the known frequency of binary stars with members hosting planetary systems and the probability for dynamical interactions between the members of such complicated systems. It would be interesting to calculate the corresponding numbers based on our models. However, we need conduct larger simulations of gravoturbulent star formation (see e.g. Klessen \& Burkert 2000, 2001, Klessen 2001a,b, Jappsen et al. 2005 ) in order to be able to derive more reliable statistics on the number of free-floating planetary-mass objects as well as the overall mass distribution of objects forming in the calculations. Such simulations are currently underway. Moreover, by using multiple levels of Particle Splitting we are now able to employ even higher numerical resolution for our discs as well as use smaller sink particles. We are thus in a position to investigate the evolution of individual discs within a realistic cluster environment at a resolution comparable to that of simulations of isolated discs. This way we can now address issues such as the amount of numerical dissipation and its effect on the angular momentum evolution of our discs.

Acknowledgments. The authors would like to thank Hans Zinnecker for many interesting discussions. SK kindly acknowledges support by an EU Commission "Marie Curie Intra-European (Individual) Fellowship" of the 6th Framework Programme. SK also acknowledges financial support by the German Research Foundation (DFG) for attending this symposium through travel grant KON 1577/2007.

\section{References}

Barnes, J. \& Hut, P. 1986, Nature, 324, 446

Bate, M. R. \& Bonnell, I. A. 2005, MNRAS, 356, 1201

Bate, M. R. \& Burkert, A. 1997, MNRAS, 288, 1060

Bate, M. R., Bonnell, I. A., \& Bromm, V. 2003, MNRAS, 339, 577

Bate, M. R., Bonnell, I. A., \& Price, N. M. 1995, MNRAS, 277, 362

Bhattal, A. S., Francis, N., Watkins, S. J., \& Whitworth, A. P. 1998, MNRAS, 297, 435

Bonnell, I. A. \& Bate, M. R. 2006, MNRAS, 370, 488

Chapman, S. J., Pongracic, H., Disney, M. J., Neslon, A. H., Turner, J. A., \& Whitworth, A. P. 1992, Nature, 359, 207

Durisen, R. H., Boss, A. P., Mayer, L., Nelson, A. F., Quinn, T., \& Rice, W. K. M. 2007, in: B. Reipurth, D. Jewitt, \& K. Keil (eds.), Protostars and Planets $V$ (Tucson: University of Arizona Press), p. 607

Heitsch, F., Burkert, A., Hartmann, L. W., Slyz, A. D., \& Devriendt, J. E. G. 2005, ApJ, 633, L113

Hennebelle, P. \& Audit, E. 2007, A\&A, 465, 431

Hunter, J. H. Jr., Sandford, M. T. II, Whitaker, R. W., \& Klein, R. I. 1986, ApJ, 305, 309

Jappsen, A.-K. \& Klessen, R. S. 2004, A\&\&A, 423, 1

Jappsen, A.-K., Klessen, R. S., Larson, R. B., Li, Y., \& Mac Low, M.-M. 2005, A\& A, 435, 611

Kitsionas, S. \& Whitworth, A. P. 2002, MMNRAS, 330, 129

Kitsionas, S. \& Whitworth, A. P. 2007, MMNRAS, 378, 507

Klessen, R. S. 2001a, ApJ, 550, L77

Klessen, R. S. 2001b, ApJ, 556, 837

Klessen, R. S. \& Burkert, A. 2000, ApJS, 128, 287

Klessen, R. S. \& Burkert, A. 2001, ApJ, 549, 386

Krumholz, M. R. \& McKee, C. F. 2005, ApJ, 630, 250

Krumholz, M. R., McKee, C. F., \& Klein, R. I. 2005, Nature, 438, 332 [astro-ph/0510412] 
Larson, R. B. 1981, MNRAS, 194, 809

Mac Low, M.-M. \& Klessen, R. S. 2004, Rev. Mod. Phys., 76, 125

Rice, W. K. M., Armitage, P. J., Bate, M. R., \& Bonnell, I. A. 2003, MNRAS, 338, 227

Schmeja, S. \& Klessen, R. S. 2004, A\&A, 419, 405

Schmeja, S. \& Klessen, R. S. 2006, A\&A, 449, 151

Shu, F. H., Adams, F. C., \& Lizano, S. 1987, ARAA, 25, 23

Stamatellos, D., Hubber, D. A., \& Whitworth, A. P. 2007, MNRAS, 382, L30

Truelove, K. J., Klein, R. I., McKee, C. F., Holliman, J. H., Howell, L. H., \& Greenough, J. A. 1997, ApJ, 489, L179

Turner, J. A., Chapman, S. J., Bhattal, A. S., Disney, M. J., Pongracic, H., \& Whitworth, A. P. 1995, MNARS, 277, 705

Vázquez-Semadeni, E., Ryu, D., Passot, T., González, R. F., \& Gazol, A. 2006, ApJ, 643, 245

Vázquez-Semadeni, E., Gómez, G. C., Jappsen, A. -K., Ballesteros-Paredes, J., González, R. F., \& Klessen, R. S. 2007, ApJ, 657, 870

Walder, R. \& Folini, D. 1998, A\&GA, 330, L21

Whitworth, A. P., Chapman, S. J., Bhattal, A. S., Disney, M. J., Pongracic, H., \& Turner, J. A. 1995, MNARS, 277, 727

Zinnecker, H. 2001, in: J. W. Menzies, \& P. D. Sackett (eds.), Microlensing 2000: A New Era of Microlensing Astrophysics, ASP Conference Proceedings, Vol. 239 (San Francisco: Astronomical Society of the Pacific), p. 223 